\title{
Magnetoencephalographic virtual recording: a novel diagnostic tool for concussion
}

\author{
Matthew Tormenti, M.D., ${ }^{1}$ Donald Krieger, Ph.D., ${ }^{1}$ Ava M. Puccio, R.N., Ph.D., ${ }^{1}$ \\ Malcolm R. McNeil, Ph.D., ${ }^{2}$ Walter Schneider, Ph.D., 3 \\ and David O. OKonkwo, M.D., Ph.D. ${ }^{1}$ \\ Departments of ${ }^{1}$ Neurological Surgery, ${ }^{2}$ Communication Science and Disorders, and ${ }^{3}$ Psychology, University \\ of Pittsburgh, Pennsylvania
}

Object. Heightened recognition of the prevalence and significance of head injury in sports and in combat veterans has brought increased attention to the physiological and behavioral consequences of concussion. Current clinical practice is in part dependent on patient self-report as the basis for medical decisions and treatment. Magnetoencephalography (MEG) shows promise in the assessment of the pathophysiological derangements in concussion. The authors have developed a novel MEG-based neuroimaging strategy to provide objective, noninvasive, diagnostic information in neurological disorders. In the current study the authors demonstrate a novel task protocol and then assess MEG virtual recordings obtained during task performance as a diagnostic tool for concussion.

Methods. Ten individuals ( 5 control volunteers and 5 patients with a history of concussion) were enrolled in this pilot study. All participants underwent an MEG evaluation during performance of a language/spatial task. Each individual produced 960 responses to 320 sentence stimuli; $0.3 \mathrm{sec}$ of MEG data from each word presentation and each response were analyzed: the data from each participant were classified using a rule constructed from the data obtained from the other 9 participants.

Results. Analysis of response times showed significant differences $\left(\mathrm{p}<10^{-4}\right)$ between concussed and normal groups, demonstrating the sensitivity of the task. The MEG measures enabled the correct classification of 8 of 10 individuals as concussed versus nonconcussed $(\mathrm{p}=0.055)$. Analysis of single-trial data classified $70 \%$ of trials correctly $\left(\mathrm{p}<10^{-10}\right)$. Concussed patients showed increased activation in the occipitoparietal and temporal regions during evaluation.

Conclusions. These pilot findings are the first evidence of the utility of MEG virtual recording in diagnosing concussion. With further refinements, MEG virtual recordings may represent a noninvasive test to diagnose concussion and monitor its resolution.

(http://thejns.org/doi/abs/10.3171/2012.10.FOCUS12282)

\section{KEY WORDS • concussion - mild traumatic brain injury • magnetoencephalography}

$\mathrm{T}$ There is no agreed-upon neuroimaging strategy to diagnose concussion. The diagnosis of concussion, which is also called MTBI, is based on patient symptom self-report and neuropsychological testing as the basis for medical decisions and the treatment paradigms. A significant percentage of patients with concussion have no abnormal findings on CT or MRI studies of the brain. ${ }^{1}$ The absence of a formal, objective neuroimaging tool to diagnose concussion reliably is a major impediment in the field.

\footnotetext{
Abbreviations used in this paper: $\mathrm{EP}=$ evoked potential; ERP = event-related potential; $\mathrm{MEG}=$ magnetoencephalography; $\mathrm{MTBI}=$ mild traumatic brain injury.
}

Magnetoencephalography shows promise for the assessment of neurophysiological derangements in neurological disorders, including TBI. This promise arises from the noninvasiveness and harmlessness of the method, its high temporal resolution, and the interpretation of results as measures of localized neurophysiological function. These properties have given hope for the development of reliable functional neuroimaging measures with which to demonstrate the mechanisms underlying the neuropsychological consequences of concussion. ${ }^{3}$ Resting MEG measures have previously enabled identification of approximately $60 \%$ of patients with concussions., ${ }^{7,910} \mathrm{~A}$ recent study ${ }^{6}$ demonstrated $85 \%$ classification accuracy.

We have developed a novel MEG-based neuroim- 
aging paradigm to provide objective, noninvasive, diagnostic neurophysiological data. In the current study, we report pilot findings obtained using MEG virtual recording as a diagnostic tool to evaluate the ability of MEG to identify individuals with concussion from healthy control volunteers. This was accomplished by asking participants to perform a task and evaluating how the magnetoencephalographic signature differs in individuals with concussion.

\section{Methods}

\section{Patient Demographic Data}

Under University of Pittsburgh Institutional Review Board approval, 10 participants (5 control volunteers and 5 patients with recent $[<4$ months $]$ concussion) were enrolled in this pilot study (Table 1). All participants were right-handed and were cognitively and neurologically normal, with no prior admission for head injury. Diagnosis of concussion was based on the patient's history and either neuropsychological testing (2 patients) or evidence of traumatic hemorrhage on head CT scans (3 patients). The control group consisted of volunteers from the general university population. Written informed consent was obtained, after which all participants underwent MEG evaluation. Either MRI (when available) or CT (1 of 10 volunteers) studies were used for anatomical localization.

\section{Task Presentation and MEG Recordings}

All participants (patients with MTBI and healthy control volunteers) were assessed using the task schematized in Fig. 1 with MEG recordings. The MEG recordings were acquired at the University of Pittsburgh Medical Center Brain Mapping Center with a 306-channel sensor array (Neuromag VectorView, Elekta AB) in a magnetically shielded room (Imdeco). The data sampling rate was $1000 \mathrm{~Hz}$, with front-end high- and low-pass filter settings of $0.1-330 \mathrm{~Hz}$.

Each trial consisted of a 5-word sentence followed by 3 consecutive test figures to which a rapid response was given; Fig. 1 shows 2 trials in the sequence. The sentences took the form "The blue square is below." The figures were either a green or blue circle or square that appeared above or below a centered fixation mark. This yielded 8 possible sentences and figures. Each word of the sentence was presented via a self-paced button press with the right index finger. When a test figure did or did not match the sentence, the participant pressed a button with the index finger or middle finger, respectively. Each participant was presented with 3205 -word sentences in 8 blocks of 40 trials each. Each sentence was followed by 3 test figures, for a grand total of 960 test figures. Each block was preceded by a brief rest, followed by task instructions. For each sentence, a 30-msec pause followed the final word before the figure appeared, and a 1000-msec pause followed the response to the third figure before the first word "The" in the next sentence appeared.

The pretest instructions included: "Respond as fast as you can without rushing and making mistakes. But if you think you have made a mistake, don't worry about it. Just go on." The instructions preceding each block of 40 trials were as follows: "Ready? Comfortable? Press the button with your index finger to fetch the words of the sentence. And use that same button when the object you are shown is as described in the sentence. If not, use the button under your middle finger."

\section{Response Time Analysis}

Self-paced reading times for each word in the sentence and response times to the test figures were analyzed using ANOVA (BMDP4V, Statistical Solutions, Inc). No significant difference in response time was found between the 2 groups. Each individuals's response time data were normalized to $\mathrm{z}$-scores (mean 0.0, SD 1.0) prior to further analysis. The factors for the ANOVA were defined as follows.

The reading times for the 2 nd-5th words were defined as 4 levels of the "Word" factor. The response times for the 3 test figures were defined as 3 levels of the "Target" factor. The blocks were defined as 8 levels of the "Block" factor. This factor was included to capture

TABLE 1: Demographic data in 10 volunteers in whom MEG virtual recording was used to assess concussion*

\begin{tabular}{|c|c|c|c|c|c|}
\hline Group \& ID No. & Age (yrs), Sex & Mechanism of Injury & Location & Initial GCS Score & CT/MRI Findings \\
\hline \multicolumn{6}{|l|}{ control } \\
\hline 123 & $26, \mathrm{M}$ & NA & NA & NA & NA \\
\hline 124 & $26, F$ & NA & NA & NA & NA \\
\hline 125 & $32, \mathrm{M}$ & NA & NA & NA & NA \\
\hline 126 & $23, \mathrm{~F}$ & NA & NA & NA & NA \\
\hline 127 & $25, \mathrm{~F}$ & NA & NA & NA & NA \\
\hline \multicolumn{6}{|l|}{ concussed } \\
\hline 122 & $24, \mathrm{M}$ & struck by a brick & rt frontal & 15 & rt frontal Fx; biconvex epidural hematoma \\
\hline 129 & $22, \mathrm{~F}$ & athletic contest & NA & 15 & none \\
\hline 131 & $16, F$ & athletic contest & NA & 15 & none \\
\hline 135 & $48, M$ & automobile & It frontal & 15 & It frontal Fx; It frontal epidural hematoma \\
\hline 140 & $57, \mathrm{M}$ & motorcycle & NA & 15 & none \\
\hline
\end{tabular}

* Fx = fracture; GCS = Glasgow Coma Scale; NA = not applicable. 


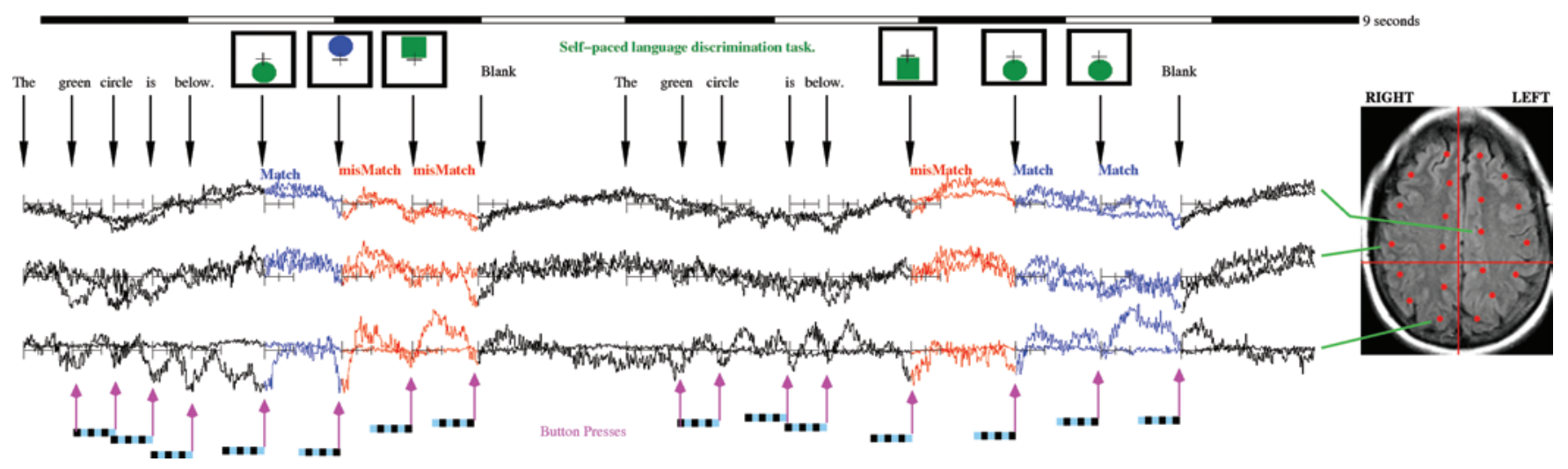

FIG. 1. Single-trial MEG virtual recordings from the locations indicated on the MRI slice obtained while this healthy volunteer performed the language/spatial task schematized in the figure and detailed above. The MEG virtual recordings show taskspecific single-trial differences for different cognitive states. The stimulus instants are shown with the black arrows; the manual responses are shown with magenta arrows. The 6 sequential 50 -msec time segments preceding each response for which the MEG virtual recordings were computed are shown at the bottom as black/blue bars (word) and blue/black bars (test figure). The lowest tracing (right posterior brain) shows responses for each word presentation, whereas the others do not. The sharp positivegoing wave just prior to the motor response for the test figures is seen in all 3 locations, but with different latencies.

changes in response time due to learning or fatigue. The 1 st, 2nd, 3rd, and 4th groups of 10 sentences within the 8 blocks were defined as 4 levels of the "wBlock" factor. This factor was included to capture so-called warm-up effects in response times within the blocks. The response times for healthy and concussed volunteers were defined as 2 levels of the "Diagnosis" factor.

Two repeated-measure ANOVAs were carried out: 1) with "Word," "Block," "wBlock," and "Diagnosis" as factors; and 2) with "Target," "Block," "wBlock," and "Diagnosis" as factors.

\section{Analysis of MEG Data}

For each participant, approximately 100 virtual electrode recording sites were selected from an anatomical scan. ${ }^{9,10}$ Magnetic resonance imaging was used in 9 participants, and CT imaging was used in 1 individual because no MR imaging was available. The virtual electrodes were located in gray matter on a sequence of axial slices $2 \mathrm{~cm}$ apart, at least $3 \mathrm{~cm}$ from the center of a sphere fit to the shape of the head. Each site was $2-3 \mathrm{~cm}$ from its nearest neighbors. Groups of virtual recording sites were divided into 7 pairs of regions. They were 6 left/ right: cerebellum, occipitoparietal, temporal, premotor, orbitofrontal, and frontal and 1 anterior/posterior: interhemispheric.

Multiple regression using the uniform conducting spherical forward solution described by Sarvas ${ }^{13}$ in 1987 was used to jointly generate the inverse solution filter pairs for the $100+$ virtual electrode recording sites. These were then used to generate single trial virtual electrode estimates for $300 \mathrm{msec}$ preceding each button press. Spectral estimates were then obtained for the $650-\mathrm{msec}$ time segments (Fig. 1) spanning these 300-msec "virtual recordings" using sine/cosine pairs. This produced estimates of the time course of the equivalent current dipole activity at each of the virtual recording sites. Stepwise linear discriminant analysis (BMDP7M, Statistical Solutions, Inc.) was applied to these estimates to construct a binary rule for identifying whether each epoch was from a concussed or healthy participant. We used 720 epochs from each of 9 participants (6480 epochs total) to construct the rule, and the rule was then applied to all 960 epochs from the 10th participant. This individual was left out of those from whom the classification rule was generated, providing an unbiased test of classification accuracy.

If at least $55 \%$ of the epochs were classified correctly $\left(\mathrm{p}<10^{-4}\right)$, the participant was counted as classified correctly. This process was repeated 10 times; for each repetition a different individual was left out when generating the rule. The person who was left out was then classified as either a patient with concussion or a healthy control.

The measures for each epoch were amplitudes of 6 frequency bands $(0-10 \mathrm{~Hz}, 10-30 \mathrm{~Hz}, \ldots, 90-110 \mathrm{~Hz})$ obtained from the 6 sequential time 50 -msec segments immediately preceding the response as described above. These signals were obtained from 7 pairs of brain regions; hence, for each epoch there were 6 frequency bands $\times 6$ time segments $\times 14$ brain regions $=504$ measures.

\section{Results}

\section{Response Time}

The mean response times for the sentence presentation showed no significant difference between concussed and healthy control volunteers ["Diagnosis" F $(1,13677)=$ $2.97, \mathrm{p}=0.085]$. Response times were then normalized to $\mathrm{z}$-scores (mean 0.0, SD 1.0) for more detailed analysis via ANOVA. Significant main effects $\left(\mathrm{p}<<10^{-4}\right)$ were found for "Word" $F(3,13677)=712.57$, "Block" $F(7,13677)=$ 236.46 , and "wBlock" $F(3,13677)=33.28$. The healthy volunteers show no change in response to the 2 nd -4 th words of the sentence, whereas those with concussion require progressively more time with each word (Fig. 2 left). Both groups show significantly longer times on the final word compared with the previous 3 words, with no difference between the groups. The reduced response times across successive trial blocks (Fig. 2 right) is consistent with learning effects, whereas the minimum response time 
M. Tormenti et al.

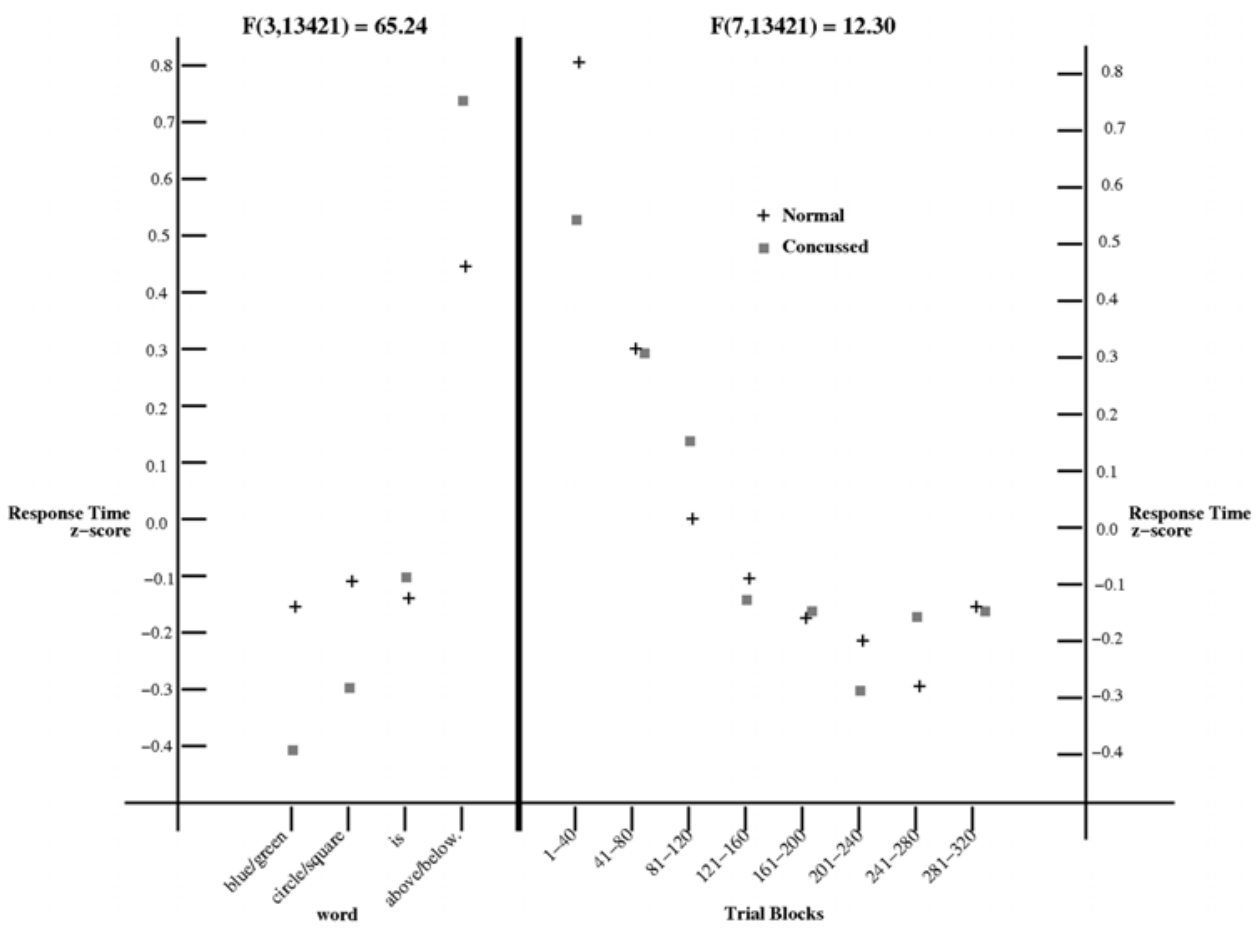

FIG. 2. Graphs showing response times to word presentation. Significant differences $\left(p<10^{-4}\right)$ in response time profiles were found for healthy versus concussed volunteers. Left: For healthy volunteers, response times were identical to all but the last word of the sentence. This difference between concussed and healthy volunteers for "word" suggests increasing mental resource allocation for those with concussion with each additional word of the sentence. Right: For healthy volunteers, response times show a minimum for the 7 th trial block versus the 6 th for those with concussion. This difference suggests earlier onset of fatigue for those with concussion.

at the 6th block for the concussed versus the 7th block for the healthy volunteers is consistent with a differential fatigue effect for the concussed compared with the healthy individuals. Differences within a block (not shown) may indicate a warm-up effect.

Significant $\left(\mathrm{p}<<10^{-4}\right)$ differences were also found between concussed and healthy participants when comparing response times for "Word" $\mathrm{F}(3,13677)=65.24$ and "Block" $F(7,13677)=12.3$. Concussed participants showed a different pattern of response time for word reading when compared with controls. Concussed patients also showed differences in learning/fatigue during presentation blocks (Fig. 2 right). A significant ( $\mathrm{p}<<$ $\left.10^{-4}\right) 3$-way interaction was found between "Diagnosis," "Block," and "wBlock" F(21,13677) = 5.63.

Response times to the figures again showed no significant main effect for "Diagnosis" $F(1,8835)=0.01$. Again, ANOVA applied to these data normalized to zscores showed significant main effects $\left(\mathrm{p} \ll<10^{-4}\right)$ for “Target" $F(2,8835)=939.43$, "Block" $F(7,8835)=57.41$, and "wBlock" $F(3,8835)=4.86$. A significant interaction ( $\mathrm{p}=0.017)$ was also found between "Diagnosis" and "Block" $F(7,8835)=2.44$, indicating that the concussed participants grew fatigued earlier than healthy volunteers.

\section{The MEG Findings}

Figure 3 shows the classification accuracies achieved by rules constructed from different regional subsets of measures. When all brain regions were used, only $35 \%$ of the epochs and 4 of the 10 participants were classified correctly. We therefore selected subsets of the brain regions for evaluation to identify which regions related to the pathological effects seen in these concussed participants. Classification accuracy for the individual epochs was used as a basis for combining regions for evaluation. The region with the highest classification accuracy was then combined with each of the other regions sequentially.

Analysis using the occipitoparietal region alone allowed for correct classification of 7 of 10 participants and correct classification of $70 \%\left(\mathrm{p} \ll 10^{-10}\right)$ of the singletrial data. As shown in the figure, no other single region produced significant classification accuracy.

The occipitoparietal region was then paired with each of the other regions. Single-trial classification accuracy was $>55 \%\left(\mathrm{p}<10^{-4}\right)$ for all pairings. All pairings except for the occipitoparietal/temporal produced a slight degradation in single-trial classification accuracy. The occipitoparietal/temporal pairing produced correct classification of $70 \%$ of trials $\left(\mathrm{p}<<10^{-10}\right)$ and 8 of 10 participants $(\mathrm{p}=0.055)$.

\section{Discussion}

The diagnosis of concussion is often difficult because symptoms may be vague or consistent with other diagnoses such as posttraumatic stress disorder. The onset of symptoms is variable and may be immediate, or symptoms may present weeks to months postinjury. ${ }^{8} \mathrm{~A}$ 

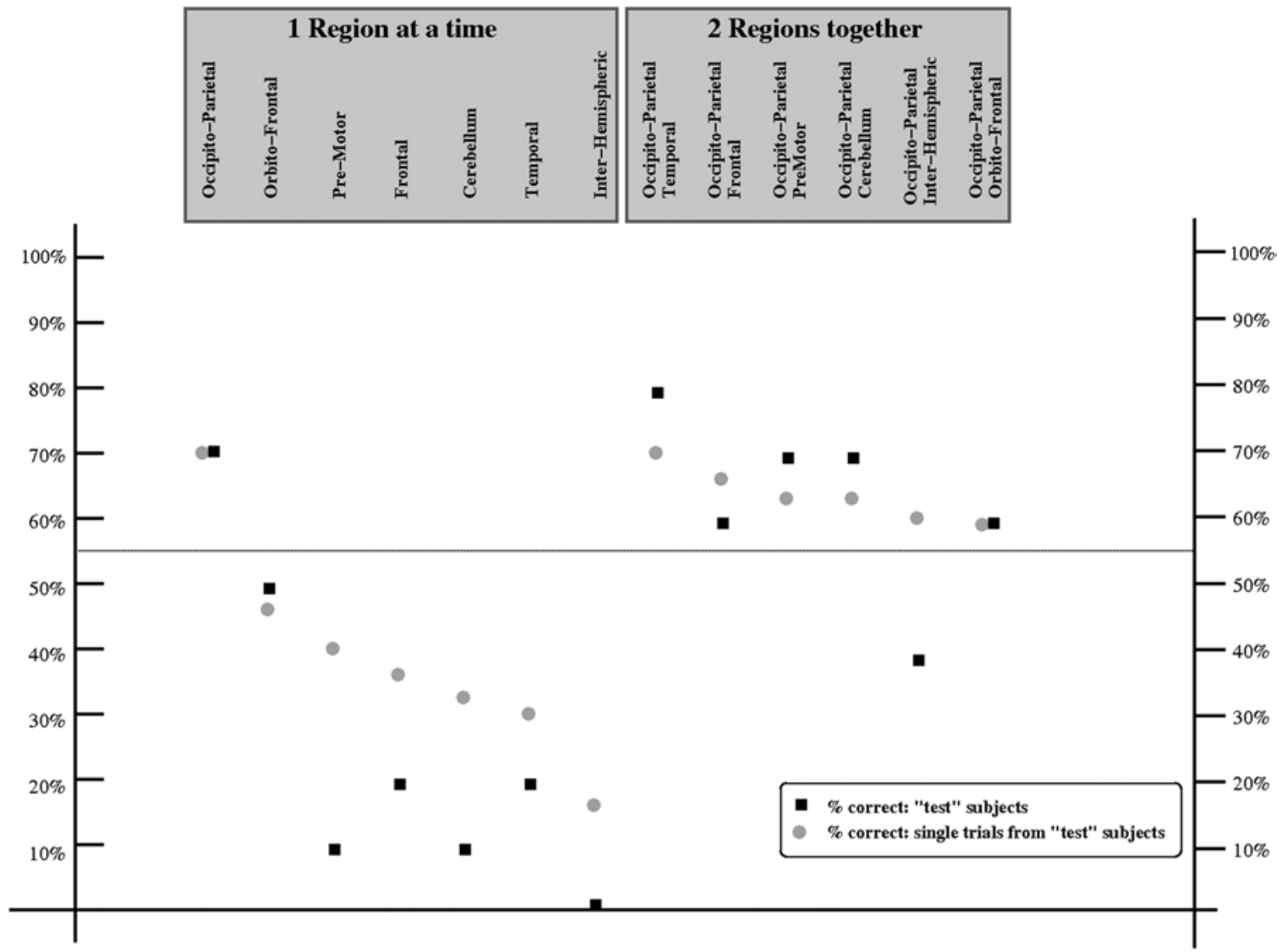

Fig. 3. A diagnostic tool. A classification rule was generated from 9 participants' single-trial data (training set). The classification rule was then applied to the trials from the 10th (left out) participant. This training/test procedure was repeated for each individual (10 times). The black squares show the number of "test" participants for whom classification accuracy exceeded $55 \%$ $\left(p<10^{-4}\right)$. The gray circles show the classification accuracy for single trials from the so-called 10th participant.

broad definition of concussion provided by the Centers for Disease Control and Prevention's MTBI Working Group includes all head injuries arising from blunt trauma or acceleration or deceleration forces with signs and symptoms of neurological dysfunction. ${ }^{1}$ These derangements include confusion, amnesia, loss of consciousness, and/or focal neurological/neuropsychological deficits. A Glasgow Coma Scale score of 13-15 classifies the injury as mild.

Once the diagnosis of concussion has been made, management approaches may be instituted. In the acute period this includes accurate documentation of the patient's symptomatology, and this may be done in the form of symptom inventory or complex neuropsychological evaluations. For sports-related concussion, many such tools have been validated and are available for use. ${ }^{11}$ This information is useful for comparison with later evaluation to assess the effects of treatment.

\section{Neurophysiological Testing and Concussion}

Evidence of electrophysiological differences following concussion has been evaluated in the past. Gaetz and Weinberg ${ }^{3}$ examined patients postconcussion with a variety of EP and ERP assessment tools. The authors found that the group of patients who reported postconcussive symptoms had a higher percentage of individuals with responses that exceeded 2.5 SDs from the mean of the norm. Using a chi-square analysis, the authors showed a statistically significant relationship between the presence of postconcussion symptoms and values for EPs and ERPs outside of the normal range.

In follow-up work to the aforementioned study, Gaetz et al. ${ }^{2}$ examined the effects of multiple concussions by dividing 60 junior hockey players into groups based on the number of concussions they had suffered. Again the authors used EP and ERP testing in the evaluation of their players. They found that athletes suffering $\geq 3$ concussions had significantly longer P3 latencies as measured by an N2/P3 paradigm using visual stimulus presentation. The P3 latency was also significantly correlated to postconcussion symptom self-reporting.

More recently, Gosselin et al. ${ }^{4}$ evaluated 20 athletes who had suffered a concussion and 10 control volunteers. These authors divided the participants with TBI into groups of 10 based on the presence or absence of postconcussive symptoms based on the Post-Concussion Symptom Scale. All athletes were evaluated using a modified auditory oddball paradigm that presents a dichotic sequence of standard and deviant tones. The authors found that athletes with concussions showed a reduction in amplitude of both early and late ERP components regardless of the presence of symptoms. 


\section{Magnetoencephalography and Concussion}

The use of MEG to study concussion is not novel. Lewine and colleagues ${ }^{9,10}$ evaluated 30 patients with postconcussive symptoms ( $>1$ year) with multimodal neuroimaging and neuropsychological evaluations. The MEG data were collected in a passive manner, with patients resting quietly with their eyes closed. The MEG study was found to be more sensitive in detecting abnormalities than SPECT or anatomical MRI. In addition, MEG was more specific for identifying patients with cognitive deficits.

The work by Lewine and coworkers ${ }^{9,10}$ was followed by a report from Huang and colleagues, ${ }^{7}$ who examined 10 concussed patients with MEG and diffusion tensor MRI. The MEG evaluations revealed several areas of slow wave activity in the concussed patients that was not found in noninjured controls. Interestingly, anatomical correlates for this slow wave activity were found on the diffusion tensor images, suggesting deafferentation of the areas that exhibited slow wave activity. Similar to the previous study, MEG data were collected with patients resting quietly. A limitation of passive collection of MEG data is that many patients with concussion may show no abnormalities at rest.

Conversely, neuropsychological behavioral measures, for example reaction time and accuracy, are quite sensitive to concussion. The MEG methodology applied in this study provides more detailed neurophysiological measures with comparable temporal resolution and with 1- to 3-cm spatial resolution.

The purpose of this study was to evaluate the utility of MEG during task performance to evaluate the effects of injury on performance and localized brain function. This was accomplished by first presenting participants with a task and determining how the brain performed that task. Comparison of task performance between healthy control and concussed volunteers allows for elucidation of the effects of injury. This approach differs from the previous studies in which MEG data was recorded passively.

The task used in this investigation yielded significant differences in response time profiles between the healthy and concussed groups and on the MEG variables, in spite of the fact that the task yielded no group differences from the group response times alone. It is noteworthy that the MEG measures from all brain regions yielded correct classification of individuals in only $35 \%$ of the epochs and in 4 of the 10 participants. We hypothesize that this poor classification accuracy was due to the presence of information within each epoch's measures to enable group classification based on features that have nothing to do with concussion per se. This is plausible given the small group sizes from which the rules were constructed.

From this analysis we set out to evaluate various combinations of regions and to limit the inclusion of information not related to brain injury. To test this and to search for potential loci of concussion, the rule construction and classification was repeated using the variables from one region at a time. The occipitoparietal region produced the highest individual region classification accuracy, and was the only single region to produce significant results.

To search further, the occipitoparietal region was paired with each of the other regions. Only in combination with the temporal region was classification accuracy improved, with 8 of 10 participants being classified correctly $(\mathrm{p}=0.055)$. Analysis of single-trial data classified $70 \%$ of trials correctly $\left(\mathrm{p}<<10^{-10}\right)$. Patients with concussions showed increased activation in occipitoparietal and temporal regions during this sentence comprehension task. Indeed, this task was chosen because it places a heavy demand on lexical-semantic processing of orthographic information and requires the integration of this sentential linguistic information with visual-spatial information to respond correctly to the sentence verification task. Large networks of linguistic and nonlinguistic information processing are required in this task, as has been shown in the Revised Token Test, ${ }^{12}$ the test from which the experimental task was derived. Indeed, this task has been shown to be sensitive to brain injury associated with both left and right hemispheres and from multiple causes, thus optimizing the opportunity for the detection of injury associated with the nonfocal damage typically found in TBI. This is especially true in the context of the MEG virtual recording used in this preliminary study. Magnetoencephalography holds promise as a tool to promote understanding, diagnosis, and assessment of treatment for concussion, particularly when it is combined with sensitive behavioral tasks that engage neural networks commonly damaged in TBI. ${ }^{5}$

\section{Caveats and Further Work}

We report results of cross-sectional pilot work with all the attendant limitations. The volunteer population was small, and the concussed volunteers comprise an inhomogeneous subset of a notoriously inhomogeneous clinical population. In addition, it is noteworthy that there are relatively few MEG facilities extant in the US and that the studies are relatively expensive. On the positive side, the setup for the MEG study used here only required 5-10 minutes, and recently developed atomic magnetometer ${ }^{14}$ "chips" hold out the hope that the cost of MEG machines may drop sharply in the future.

The strength of both the behavioral and neurophysiological findings hold out hope that this approach may lead to objective diagnostic measures applicable to individual patients as well as individualized functional localization of regional brain pathology. To pursue this, these results must hold up for a larger patient population and must be demonstrated in a prospective longitudinal design. To this end, a cohort study may be justified in which baseline and follow-up MEG studies are obtained from a high-risk but healthy group; for example, a high school football squad or college female cheerleaders. For this study, those in the high-risk group who do not sustain concussions will serve as controls for those who do, along with the individual control inherent in the longitudinal design.

\section{Disclosure}

This work was supported by Copeland funding from the Pittsburgh Foundation. The authors report no conflict of interest concerning the materials or methods used in this study or the findings specified in this paper.

Author contributions to the study and manuscript prepara- 


\section{Use of MEG virtual recordings in diagnosis of concussion}

tion include the following. Conception and design: Krieger, Tormenti, Puccio, McNeil, Okonkwo. Acquisition of data: Krieger. Analysis and interpretation of data: all authors. Drafting the article: Krieger, Tormenti, McNeil, Okonkwo. Critically revising the article: all authors. Reviewed submitted version of manuscript: Tormenti, McNeil, Schneider, Okonkwo. Statistical analysis: Krieger. Administrative/technical/material support: Krieger, Tormenti, Okonkwo. Study supervision: Krieger, Tormenti, Puccio. Principal Investigator for funding source: Tormenti, Puccio.

\section{References}

1. Centers for Disease Control: Heads Up: Facts for Physicians about Mild Traumatic Brain Injury (MTBI). (http://www. cdc.gov/ncipc/pub-res/tbi_toolkit/physicians/mtbi/mtbi.pdf) [Accessed October 19, 2012]

2. Gaetz M, Goodman D, Weinberg H: Electrophysiological evidence for the cumulative effects of concussion. Brain Inj 14:1077-1088, 2000

3. Gaetz M, Weinberg H: Electrophysiological indices of persistent post-concussion symptoms. Brain Inj 14:815-832, 2000

4. Gosselin N, Thériault M, Leclerc S, Montplaisir J, Lassonde M: Neurophysiological anomalies in symptomatic and asymptomatic concussed athletes. Neurosurgery 58:1151-1161, 2006

5. Hinchliffe FJ, Murdoch BE, Chenery HJ: Towards a conceptualization of language and cognitive impairment in closedhead injury: use of clinical measures. Brain Inj 12:109-132, 1998

6. Huang MX, Nichols S, Robb A, Angeles A, Drake A, Holland M, et al: An automatic MEG low-frequency source imaging approach for detecting injuries in mild and moderate TBI patients with blast and non-blast causes. Neuroimage 61:1067-1082, 2012

7. Huang MX, Theilmann RJ, Robb A, Angeles A, Nichols S, Drake A, et al: Integrated imaging approach with MEG and
DTI to detect mild traumatic brain injury in military and civilian patients. J Neurotrauma 26:1213-1226, 2009

8. Kushner D: Mild traumatic brain injury: toward understanding manifestations and treatment. Arch Intern Med 158: 1617-1624, 1998

9. Lewine JD, Davis JT, Bigler ED, Thoma R, Hill D, Funke M, et al: Objective documentation of traumatic brain injury subsequent to mild head trauma: multimodal brain imaging with MEG, SPECT, and MRI. J Head Trauma Rehabil 22:141155,2007

10. Lewine JD, Davis JT, Sloan JH, Kodituwakku PW, Orrison WW Jr: Neuromagnetic assessment of pathophysiologic brain activity induced by minor head trauma. AJNR Am J Neuroradiol 20:857-866, 1999

11. Maroon JC, Lovell MR, Norwig J, Podell K, Powell JW, Hartl R: Cerebral concussion in athletes: evaluation and neuropsychological testing. Neurosurgery 47:659-672, 2000

12. McNeil MR, Prescott TE: Revised Token Test. Austin, TX: Pro-Ed, 1978

13. Sarvas J: Basic mathematical and electromagnetic concepts of the biomagnetic inverse problem. Phys Med Biol 32:11-22, 1987

14. Xia H, Baranga AB, Hoffman D, Romalis MV: Magnetoencephalography with an atomic magnetometer. Appl Phys Lett 89:211104, 2006

Manuscript submitted August 13, 2012.

Accepted October 10, 2012.

Please include this information when citing this paper: DOI: 10.3171/2012.10.FOCUS12282.

Address correspondence to: Donald Krieger, Ph.D., Department of Neurological Surgery, UPMC Presbyterian, Suite B400, 200 Lothrop Street, Pittsburgh, Pennsylvania 15213-2582. email: kriegerd@upmc. edu. 